OPEN ACCESS

Edited by: Jianxun Ding,

Chinese Academy of Sciences, China

Reviewed by:

Evelien Smits,

University of Antwerp, Belgium

Xiangru Feng,

Chinese Academy of Sciences, China

${ }^{*}$ Correspondence:

Hong Cui

Cuihong2017@126.com

Specialty section: This article was submitted to Experimental Pharmacology and Drug Discovery,

a section of the journal

Frontiers in Pharmacology

Received: 17 February 2020 Accepted: 12 June 2020

Published: 30 June 2020

Citation:

Qian C, Yang L-J and Cui H (2020)

Recent Advances in Nanotechnology

for Dendritic Cell-Based Immunotherapy.

Front. Pharmacol. 11:960. doi: 10.3389/fphar.2020.00960
Recent Advances

\section{in Nanotechnology for Dendritic Cell-Based Immunotherapy}

\author{
Chen Qian, Li-Jun Yang and Hong Cui* \\ Department of Pediatrics, Beijing Friendship Hospital, Capital Medical University, Beijing, China
}

Dendritic cells (DCs) are the most important antigen-presenting cells that determine cancer immune responses by regulating immune activation and tolerance, especially in the initiation stage of specific responses. Manipulation of DCs to enhance specific antitumor immune response is considered to be a powerful tool for tumor eradication. Nanotechnology, which can incorporate multifunction components and show spatiotemporal control properties, is of great interest and is widely investigated for its ability to improve immune response activity against cancer and even for prevention and avoiding recurrence. In this mini-review, we aim to provide a general view of DC-based immunotherapy, including that involving the promising nanotechnology. Particularly we discuss: (1) manipulation or engineering of DCs for adoptive vaccination, (2) employing DCs as a combination to more existing therapeutics in tumor treatment, and (3) direct modulation of DCs in vivo to enhance antigen presentation efficacy and priming $T$ cells subsequently. We comprehensively discuss the updates on the application of nanotechnology in DC-based immunotherapy and provide some insights on the challenges and opportunities of DC-based immunotherapeutics, including the potential of nanotechnology, against cancers.

Keywords: nanotechnology, dendritic cell, cancer, immunotherapy, antigen delivery, cross-presentation

\section{INTRODUCTION}

Cancer therapies have been evolving with advances in oncology (Couzin-Frankel, 2013). There has been some success with the use of traditional cancer therapies such as surgery (Krook et al., 1991; Wolf, 1991; Macdonald et al., 2001; Coffey et al., 2003), chemotherapy (Liu et al., 2011; Al-Lazikani et al., 2012; Koppelmans et al., 2012; Chen et al., 2017), and radiotherapy (Sauer et al., 2004; Abe et al., 2005). However, low-specificity, drug resistance, and side effects hinder the efficacies of these tumor treatments (Lippert et al., 2008; Guth et al., 2011; Housman et al., 2014; Rothermundt, 2015; Leary et al., 2018). Inducing or boosting up the patients' own immune responses enable specific recognition and killing of tumor cells. Besides, immune response-mediated tumor eradication reduces the potential risks of toxicity and drug resistance (Beltran et al., 2011; Zhong et al., 2017). Therefore, in recent years, cancer immunotherapy is burgeoning and is considered to be the fourth important therapeutic method to deal with cancers. For example, the first tumor vaccine Sipuleucel$\mathrm{T}$ was approved by the U.S. Food and Drug Administration (FDA) in 2010 for prostate cancer treatment (Higano et al., 2009; Kantoff et al., 2010). In the next year, the first immune-checkpoint 
inhibitor antibody Ipilimumab (anti-CTLA4 antibody) was approved for the treatment of advanced melanoma (Robert et al., 2011). In the next few years, multiple immunecheckpoint blockage antibodies were approved in various cancers and achieved great success (Brahmer et al., 2015; Le et al., 2015; Reck et al., 2016). Encouragingly, the Nobel prize in physiology and medicine honored James P. Allison and Tasuku Honjo for their discovery of the efficacy of inhibiting negative immune regulation in cancer therapy. Additionally, Tisagenlecleucel, the first chimeric antigen receptor- $T$ cellbased adoptive transfer treatment for pediatric and young adult acute lymphoblastic leukemia and adult diffuse large Bcell lymphoma was approved in 2018 by the FDA (June et al., 2018; Maude et al., 2018).

Although great achievements have been made in cancer immunotherapy, only some percentage of patients can benefit from those promising immunotherapeutics, mainly owing to the immunosuppressive microenvironment of the solid tumor and immune tolerance to mono-therapeutics (Albini et al., 2018; Binnewies et al., 2018; Costa et al., 2018; Feng et al., 2018; Knudson et al., 2018; Zhao et al., 2018). Therefore, maximizing the potential and ability of the immune system to overcome tumors is critical (Zhang and Bevan, 2011; Nicholson, 2016). Dendritic cells (DCs) are the initiators of specific immune responses, and when activated effectively can lead to priming of $\mathrm{T}$ cells to elicit immune antitumor responses for tumor destruction (Rescigno et al., 1997; Leifer, 2017). More importantly, results of whole exome sequencing and RNA sequencing present the possibility of developing DC-targeting vaccines and DC-based immunotherapy (Leitner et al., 1999; Mathan et al., 2017). Manipulating DCs by taking advantage of the controllable and modifiable features of nanotechnology shows promising antitumor responses both in vitro and in vivo.

\section{DCS IN IMMUNE SYSTEM}

DCs are originated from dedicated hematopoietic precursor cells and transformed into DC in variable stimuli in physiological environment. (Thomas and Lipsky, 1996; Ardavin et al., 2001; Ardavin, 2003; Wang et al., 2019b). Circulating blood cells such as monocytes, white blood cells may differentiate to matured DCs owing to the crosstalk among diverse signals coordination (Rutella et al., 2006; Williams et al., 2013). DCs were demonstrated to play a crucial role in mediating innate and adaptive immune responses in 1970 s by Ralph Steinman and Zanvil A. Cohn, who were honored with the Noble Prize for their discovery. Since then, DCs have been documented as the most effective antigen-presenting cells that activate primary and subsequent memory immune responses (Kadowaki, 2009).

DCs as antigen-presenting cells encounter antigens and present them to $\mathrm{T}$ cell for priming in the form of antigen peptide-major histocompatibility complex (MHC) (Cohen et al., 2003; Zehn et al., 2004). Antigens may circulate through peripheral blood and stimulate DCs or captured by tissue resident DCs such as lymph node, spleen. Whatever, DCs' received antigens are required to migrate to the lymphoid organs which determines the subsequent $\mathrm{T}$ cell activation efficacy due to the abundance of $\mathrm{T}$ cells in the lymphoid organs (Mempel et al., 2004) (Segura and Villadangos, 2009). The success of DCs' activation requires two signals, antigens and activation stimuli. The second activation signals may be provided exogenously such as by lipopolysaccharide (Morrison and Kline, 1977), Toll-like receptor (TLR) ligands (Kawai and Akira, 2007), and antibodies targeting activation of receptors like tumor necrosis factor (Schnurr et al., 2000), proinflammatory cytokines IFN- $\gamma$, etc (Simmons et al., 2012; Minton, 2014). Endogenous damage-associated molecular patterns such as high mobility group proteins (Raucci et al., 2007), calreticulin (Li et al., 2015), and heat shock proteins with immunogenic features are also capable of maturing DCs (Bethke et al., 2002). Similarly, two signals are required for $\mathrm{T}$ cell priming; one is a specific antigen-MHC I/II complex and the other one is a costimulatory signal expressed on activated DCs. mDCs with antigen-MHC complex expression induce $\mathrm{T}$ cells to differentiate into Th1 or Th2 cells under the condition of variable cytokines, for example, IL-12, which is essential to activate cytotoxic T cells. In some other cases, under the condition of IL-4, it may switch immunity with antibody secretion. Mostly, these activated CD8+ $\mathrm{T}$ cells are fully functional, with cytotoxicity and ability to secrete IFN- $\gamma$ for highly effective and specific killing of cancer cells. Other than cytotoxic T cell stimulation, matured DCs (mDCs) may also function to neutralize antibody secretion by B cells in the way of IL-10 and IL-33 secretion, driving Th2 immunity and influencing immunoglobulin subtype polarization (Segura et al., 2013). Additionally, DCs with inflammatory features induce Th17 differentiation, which promotes cytotoxic $\mathrm{T}$ cell responses and regresses tumor (Murugaiyan and Saha, 2009; Segura et al., 2013; Guéry and Hugues, 2015). Apart from stimulating specific adaptive immunity, $\mathrm{mDCs}$ can also be decorated with IL-12, IL-15, and type I IFNs which are positive to NK cell functions in innate immunity. Thus, the DCs act as a bridge between innate and adaptive immunity for host defenses (Figure 1). As discussed above, the immature DCs that are usually found in peripheral lymphoid tissues process antigens without activation stimuli and are capable of presenting antigen-MHC to naïve $\mathrm{T}$ cells, resulting in tolerance to $\mathrm{T}$ cell responses (Probst et al., 2005).

\section{MANIPULATION OF DCS FOR VACCINATION}

DCs that initiate adaptive immune responses, including antitumorigenesis, are of great interest worldwide, owing to their ability to be able to present tumor-associated antigens and prime subsequent antitumor responses. Because of their critical role with professional presentation expertise in the immune system, exploiting DC for vaccination is considered to provide a powerful tool to prevent and cure infections and cancers (Lizotte et al., 2016; Roden and Stern, 2018; Wang et al., 2019a; Wen et al., 2019). As initiators of adaptive 


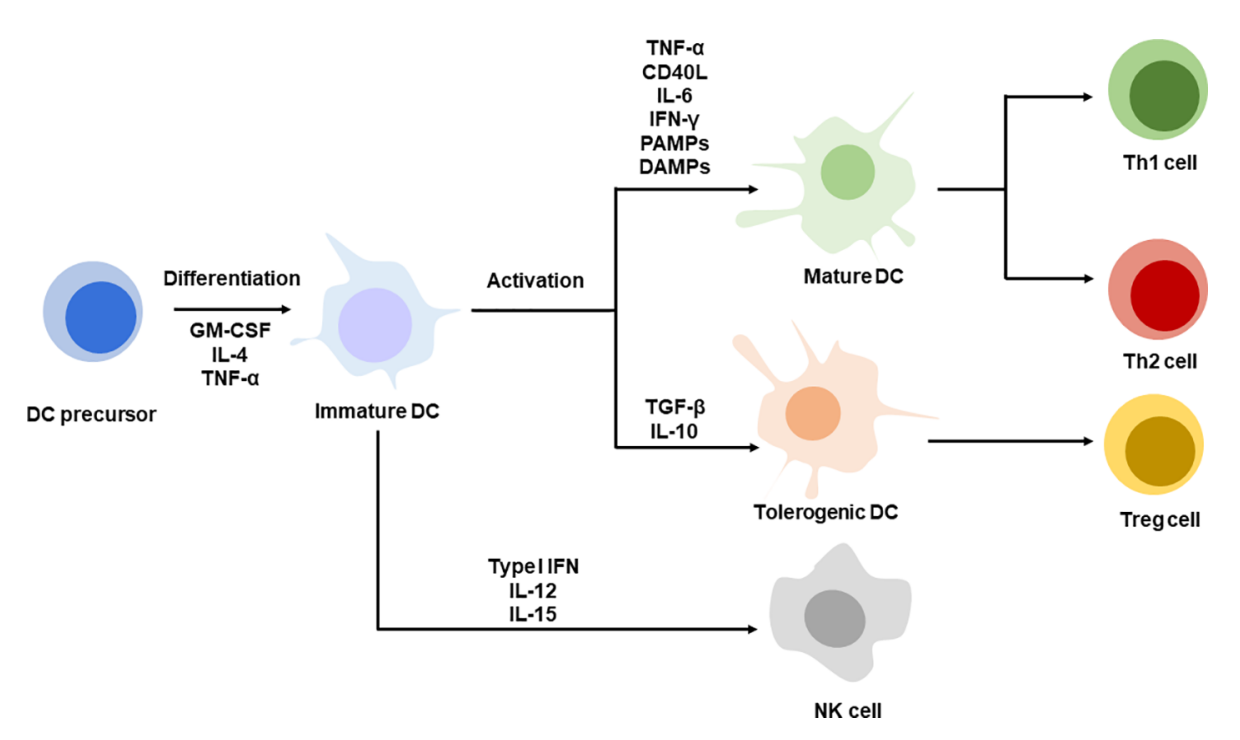

FIGURE 1 | DC differentiation and maturation. DC precursors are differentiating into immature DC. Immature DCs are activated with specific stimuli, TNF- $\alpha$, CD4OL, IL-6, IFN- $\gamma$, PAMPs, DAMPs promote DC maturation, corresponding to Th1 or Th2 cells priming. DCs stimulate with TGF- $\beta$, IL-10 present tolerogenic phenotype leading to Treg differentiation. Immature DC decorated with type I IFN, IL-12 and IL-15 favor NK cell function.

immunity, DCs are ideal targets for ex vivo education and adoptive vaccination, which can induce specific antitumor immune responses in patients in vivo (Lövgren et al., 2018). Conventional techniques to develop DC-based vaccines involved isolation or culture precursor cells from patients' peripheral blood, loading them with antigens in vitro and applying certain maturation stimuli to promote DC maturation. Precursor cells such as CD34+ hematopoietic cells, monocytes cultured with certain stimuli enabled differentiating into immature DCs. In order to differentiate and expand DCs in vitro, immature DCs are loaded with tumor antigens such as tumor lysates, peptides, proteins, nucleotides, or fused with tumor cells, under the condition of stimulatory molecules. Challenges remained although more techniques have been developed. Peptides can be loaded directly on MHC molecules; however, it requires clear and definite information not only on the epitopes such as sequence and conserved motif, but also on an individual's HLA configuration that determines the type of immune responses and adaptive immunity. Clinical study is ongoing to confirm the immunogenicity of peptide-loaded DC vaccination (NCT02334735). Instead of peptides, loading of proteins or lysates has also been used for numerous cancer treatments (NCT00045968). Although these require further intracellular processing, the major advantage of using whole protein processing is its potential to induce both CD4+ and CD8+ T cell responses (Constantino et al., 2016; Wang et al., 2017; Sharma et al., 2018). To realize the DC function of initiating specific antitumor immunity, DCs loaded with tumor-associated antigens require stimuli (example, CD40L or TLR agonist) for maturation. The mDCs are then transferred back to the patients to mediate specific immune response. The whole process is complicated and requires skilled operation and high cost. Most clinical studies on DC vaccines are in combination with chemotherapy (NCT03688178, NCT03657966, NCT03047525) (Kongsted et al., 2017), radiotherapy (NCT03226236,), targeted agents (Ogasawara et al., 2018; Palma et al., 2018), or immunotherapeutic regimens (NCT03546426, NCT03735290, NCT03450044, NCT03735589). Clinical studies implied that technologies that combine antigen loading and various combination agents are required and of great potential.

Nanotechnology refers to the technology utilizing nanosized materials that range from 10 to $100 \mathrm{~nm}$ in size; these are emerging as an ideal tool for cancer therapy. Nanomaterials with divergent compositions enable passive or active targeting, which enhances the efficacy and reduces the toxicity of treatment (Liu et al., 2018; Wang and Mooney, 2018; Feng et al., 2019). The ability of DCs to capture, process, and present antigen to induce $\mathrm{T}$ cell priming is essential in adaptive antitumor immunity. Therefore, nanomaterials targeting DCs are considered to be a promising tool to boost an efficient and specific anticancer immune response. Nanomaterials also incorporate multifunctional molecules together to modulate part or whole of the antigen presentation (Parvanian et al., 2017; Sangtani et al., 2017). Functional nanomaterials with lysosome escaping ability have been widely reported; these nanomaterials are favorable for antigen delivery to the cytoplasm and their intracellular digestion, which further contributes to antigen processing and presentation (Jiang et al., 2018; Zhong et al., 2019). Lipid-based nanoparticles and polymeric nanoparticles (Reichmuth et al., 2016; Zhou et al., 2016; Gulla et al., 2019; Wen et al., 2019; Lin et al., 2020), with better biocompatibility, have been reported in the development of DC targeted vaccines for anticancer and antiviral treatments. Liposome-based nanoparticles containing molecules such as trivalent influenza antigen, OVA, and heat shock 
proteins, have been investigated for their ability to activate the immune system in disease treatment. Delivery of protein, peptide, and nucleic acid antigens, which can induce corresponding immune responses, to DCs via nanomaterials might probably increase their circulation and reduce their degradation in vivo, which are necessary for future approaches that aim to directly activate DCs in vivo (Rogel et al., 1985; Paglia et al., 1996; Ghadersohi and Sood, 2001; Goodwin and Huang, 2017; Wongso et al., 2017; Dellacherie et al., 2018). Moreover, nanomaterials (e.g., gold, aluminum nanoparticles) probably enhance internalization at the single cell level (Wang et al., 2015; Li et al., 2016; Wang et al., 2016). Aluminum, which was documented as an adjuvant for Th2 stimulation, failed to generate CD8+ T cell responses (Ho et al., 2018). However, using nanosized aluminum particles $(100 \mathrm{~nm})$ stabilized by PEGcontaining polymer showed higher internalization in antigenpresenting cells and activated CD8+ $\mathrm{T}$ cells for curing cancer. Thus, nanotechnology not only improves the bio- or physiochemical features of antigen formulation through antigen protection, quantity improvement, function combination, and antigen presentation pathway, but also redefines the efficacy of traditional agents or materials with potential novel functions in antitumor immunity modulation (Zupančič et al., 2017; Smith et al., 2018; Zhong et al., 2019), which are all beneficial for the subsequent adaptive immunity against cancers. Importantly, a few cases that used nanotechnology for DC vaccination have been reported. For instance, liposome based Tecemotide, AS15, DepoVax, decorating with tumor-associated antigens are in clinical trials (Berinstein et al., 2012; Butts et al., 2014; Vansteenkiste et al., 2014). Virus-like nanoparticles CYT004-MelQbG10 delivering melanomaassociated antigen peptide are in Phase II clinical trials (Speiser et al., 2010). These pre/clinical trials evidenced the potential targeting DC for antitumor immunotherapy.

\section{IN VIVO ACTIVATION OF DC FOR ENHANCING ANTIGEN PRESENTATION}

The traditional approach of activating DCs in vitro and transferring them back into patients is expensive, labordependent, and difficult to evaluate antitumor immunity generation parallelly in patients. Targeting DCs for in vivo activation with antitumor immunity enhancement is another promising DC-based therapeutic strategy to induce specific antitumor immune responses; this approach directly activates DCs in vivo and can potentially generate a large number of antitumor responses. Nanotechnology-enabled spatiotemporal delivery of formulations aimed to directly activate DCs has been demonstrated in numerous cases (Figure 2). These formulations can be classified into three categories: (1) those that target and activate local DC response in the tumor microenvironment in addition to chemotherapy/radiotherapy (Sau et al., 2018); (2) those that target and activate lymph node-resident DCs, and in addition, target activation receptors on DCs, thereby boosting specific $\mathrm{T}$ cell response (De Koker et al., 2016; Jiang et al., 2017); and (3) those that modulate intracellular antigen presentation process for higher crosspresentation efficacy (Sil et al., 2019; Wang et al., 2019a).

\section{DC-Based Combination Therapy}

Because of the heterogeneous features of variable tumors and their immune microenvironment, "cold" tumors with low immunogenicity show enrichment of immunosuppressive cells such as macrophages and myeloid-derived suppressor cells around the microenvironment to escape from the immune surveillance (Smyth et al., 2006; Dadi et al., 2016; Lussier and Schreiber, 2016; Haanen, 2017). Therefore, combination therapies aimed to change the tumor immunogenic phenotype

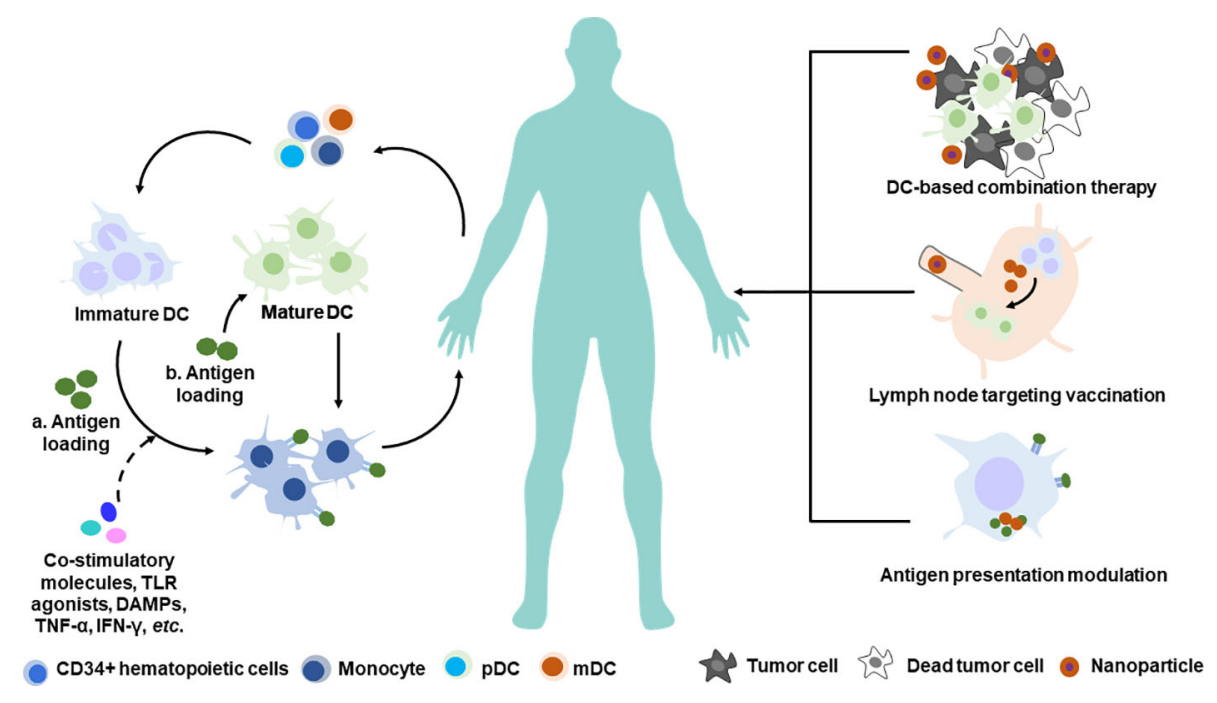

FIGURE 2 | Nanotechnology-based manipulation of DCs therapy. Conventional strategy of DC-based adoptive transfer therapy (left) and novel strategy employing nanotechnology for future DC-based immunotherapy (right). a. Antigen loading on immature DC in the scenario of DC maturation stimuli. b. Antigen loading on mature DC directly. pDC, plasmacytoid dendritic cell; mDC, myeloid dendritic cell. 
provide a means to reduce the mortality associated with cancers (Da Silva et al., 2016; Nam et al., 2019). Chemo-immunotherapy approach that incorporates doxorubicin (DOX) and indoximod (IND) with phospholipid as a prodrug shows improved pharmacokinetics and accumulation in 4T1 breast tumor model. In such an approach, the chemo drugs induce tumor cell death, eliciting immunogenic reactions along with the debris being taken up by DCs, leading to further antigen presentation and naïve $\mathrm{T}$ cell priming. The activated $\mathrm{T}$ cells present cytotoxic effects on tumor cells through perforin and release of IFN- $\gamma$ for robust killing of both primary and metastatic tumors. Not only does the dual-functional liposomes exert synergistic antitumor effects via DOX and IND components, but also the immunecheckpoint blockage combination treatment which further boosts immune responses allowing metastatic tumor eradication (Johnson et al., 2017; Lu et al., 2018). Radiotherapy is also known to promote CD8+ $\mathrm{T}$ cells around the tumor, however, radiation-induced immunosuppression may lead to treatment failure. Bismuth sulfide nanoparticles that conjugate immunoactive polysaccharide can increase radiotherapy sensitivity and activate DCs. Meanwhile, the nanoparticle can further enhance DC maturation and their distribution in tumor. Several studies have indicated that photodynamic therapy, which induces tumor cell lysates, is usually accompanied with damageassociated molecular pattern release that promotes DC activation, leading to specific antitumor immunity. Formulating photosensitizer is a major challenge in photodynamic therapy and further acceptance in clinic. The application of nanotechnology is a stride forward in solving this problem to some extent (Lucky et al., 2015), which favors photodynamic and DC combination therapy (Chen et al., 2016; Yu et al., 2019).

\section{Lymph Node Targeting Vaccination}

Peripheral DCs that encounter antigens are required to be trafficked to the lymph node where abundant lymphocytes are located and enabled highly efficient $\mathrm{T}$ cell priming. Moreover, diverse cytokines in the lymph node further promote DC maturation and exert function (Manfredi et al., 2008; MartínFontecha et al., 2009; Granot et al., 2017). Modifying antigens to target the lymph node and enhance lymph node-resident DC uptake was demonstrated to be a promising strategy in vaccine designing to initiate effective immune responses (Van Herck et al., 2018). Developing delivery systems with variable size can greatly influence the lymph node trafficking and DC uptake of antigens (Bachmann and Jennings, 2010; Kuai et al., 2017; Zhang et al., 2018). Proteins or subunit viral antigens within $10 \mathrm{~nm}$ are mostly incorporated with adjuvants to form larger-sized particles or aggregates. Supramolecular antigen formulations such as virus-like particles range from 20 to $200 \mathrm{~nm}$. Virosomes with liposomes and antigens are approximately 100-200 nm (Jennings and Bachmann, 2007; Bachmann and Jennings, 2010). These antigen formulations are presented in the form of nano- or microparticles. Zhang et al. (2018) systemically investigated the influence of size and charge on the micelle-like formulations and their contributions to modulating immune responses, including lymph node accumulation, and DC internalization and immunogenicity, which provide an insight into the micelles and the generated immune responses. Lymph node accumulation and interaction properties of antigen-presenting cells are the two most important factors that influence the immunogenicity of nanoparticulated/nanoformulated antigens. Lymph node draining and DC uptake capability are both sizedependent processes, with a diameter of 10-200 nm considered to be optimal to stimulate immune response. Additionally, the authors claimed that the optimized size range was relatively independent of the materials, as the efficacy of lymph node draining and DC internalization is critically attributed to the particle size. However, variable materials with surface charges might influence the internalization capability of the antigen-presenting cells. Micelles with positive charged surface are beneficial for the enhancement of host antibody production (Reddy et al., 2007; Irvine et al., 2013). The previously published reviews provide an overview of materials on physiochemistry properties of antigen formulation in exerting effective immune responses.

Zhang et al. (2019) designed and synthesized a hybrid nanoparticle including the TLR 7/8 agonist imiquimod, TLR4 agonist monophosphoryl lipid A, polycaprolactone-polyethylenepolycaprolactone copolymer, dioleoyl-3-trimethylammonium propane, and 1,2-Distearoyl-sn-Glycero-3-Phosphoethanolaminemannos. The hybrid nanoparticles enabled trafficking to secondary lymphoid tissues, spatiotemporal delivery and stimulation of both extracellular and intracellular TLRs, which was critical for efficient DC activation. It was demonstrated that the hybrid nanoparticles favorably enhanced the DC uptake of tumor antigen and cytokine secretion by mDCs. Additionally, homogenous tumor cells reinoculated into C57BL/6 mice were inhibited upon treatment with the hybrid nanoparticles, highlighting the promising specific antitumor immune responses mediated by the nanoparticles.

\section{Modulating Antigen Presentation for DC Vaccination}

Antigen processing is an essential process for the final antigen presentation efficacy followed by lymph node draining accumulation and uptake by antigen-presenting cells. Thus, regulating intracellular antigen processing is important for antigen presentation and subsequent $\mathrm{T}$ cell priming. DCs are considered with well capacity for lysosomal proteolysis. It was documented that macrophages containing abundant lysosomal proteases enabled internalized protein degradation rapidly. In contrast, DCs with limited lysosomal capacity due to poor proteolysis degraded antigens slowly in vivo thus preserved antigen for an extended period. The limited proteolysis capacity benefits antigen presentation (Delamarre et al., 2005). Moderate lysosomal capacity of DCs favored antigen presentation implied us the opportunity of other pathway modulation in enhancing antigen degradation and presentation. It has been reported that inducing autophagy, an intracellular degradation and clearance process of unnecessary or dysfunctional components, is beneficial for antigen processing by DCs and leads to highly effective $\mathrm{T}$ cell stimulation. Wang et. al. combined a model antigen peptide $\mathrm{OVA}_{257-264}$, autophagy inducing peptide Beclin1, and a $\mathrm{pH}$ sensitive polymer together by covalent 
conjugation and fabricated an autophagy-inducing nanoactivator, which could induce autophagy in DCs, and evaluated the antigenspecific immune responses both in vitro and in vivo (Wang et al., 2015; Wang et al., 2019a). They demonstrated that induction of autophagy improved the antigen-presenting efficacy and $\mathrm{T}$ cell activation. Notably, the MHC I-OVA $257-264$ complex expression was significantly decreased on combining the nanoactivator with an autophagy inhibitor. Furthermore, cross-presentation DCs and antigen-specific $\mathrm{T}$ cells increased, along with the appearance of specific antitumor immune responses, tumor infiltrated cytotoxic $\mathrm{T}$ cell accumulation, and tumor growth inhibition, indicating that the nanoactivators were capable of initiating antigen-specific immune responses in vivo for the eradication of established solid tumors.

\section{CONCLUSION}

Considering that DCs play a vital role in priming naïve $\mathrm{T}$ cell and inducing adaptive immunity, tremendous efforts have been made in manipulating DC for cancer immunotherapy. It is documented that adoptive DC transfer can elicit specific immune responses in approximately $70 \%$ patients (Draube et al., 2011). Controlled parallel studies and comparative analysis are limited but essential for outcome evaluation and further clinical translation of adoptive DC vaccination. Moreover, immunosuppressive patients and intrinsic tumorassociated factors such as inhibitory ligands and Tregs do not favorably respond to DC-based immunotherapy. Hence, there is a need to improve therapeutic efficacy by manipulating DCs independently in the primary step. Developing combination strategies are expected to be promising in the exploitation of DC vaccines (Kalinski et al., 2013). Taking the advantages of nanotechnology, manipulation, and engineering of DCs in vitro can be simplified and its activation efficiency can be improved, which could increase the therapeutic efficacy of adoptive DC transfer treatment. In addition, nano-engineering can allow in

\section{REFERENCES}

Abe, O., Abe, R., Enomoto, K., Kikuchi, K., Koyama, H., Masuda, H., et al. (2005). Effects of radiotherapy and of differences in the extent of surgery for early breast cancer on local recurrence and 15-year survival: an overview of the randomised trials. Lancet 366, 2087-2106. doi: 10.1016/S0140-6736(05)67887-7

Albini, A., Bruno, A., Noonan, D. M., and Mortara, L. (2018). Contribution to Tumor Angiogenesis From Innate Immune Cells Within the Tumor Microenvironment: Implications for Immunotherapy. Front. Immunol. 9, 1-9. doi: 10.3389/fimmu.2018.00527

Al-Lazikani, B., Banerji, U., and Workman, P. (2012). Combinatorial drug therapy for cancer in the post-genomic era. Nat. Biotechnol. 30, 679-691. doi: 10.1038/ nbt. 2284

Ardavin, C., Del Hoyo, G. M., Martin, P., Anjuere, F., Arias, C. F., Marin, A. R., et al. (2001). Origin and differentiation of dendritic cells. Trends Immunol. 22, 691-700. doi: 10.1016/S1471-4906(01)02059-2

Ardavin, C. (2003). Origin, precursors and differentiation of mouse dendritic cells. Nat. Rev. Immunol. 3, 582-590. doi: 10.1038/nri1127

Bachmann, M. F., and Jennings, G. T. (2010). Vaccine delivery: a matter of size, geometry, kinetics and molecular patterns. Nat. Rev. Immunol. 10, 787-796. doi: $10.1038 /$ nri2868 vivo DC activation, mimicking natural antigen presentation followed by priming processes. Directly activating DCs in vivo fully employed the moderate lysosomal proteolysis feature that degraded antigens slowly which enabled sustaining antigen presentation. Making use of nanotechnology can also allow the conjugation of DCs to extraordinary functional molecules, which can further enhance antigen presentation, mediating sophisticated crosstalk inside the antigen-presenting cells and or work together with other cells, thereby contributing to the efficacy of DC-based immunotherapy. Another challenge to conventional DC-based therapeutics is that activated DCs are required to migrate to lymph nodes and prime $\mathrm{T}$ cells for specific antitumor responses; however, the majority of the DCs are unable to reach the lymph nodes, leading to limited specific immune responses. In contrast, nanoparticles enable higher lymph node accumulation, contributing to highly effective lymph node draining. Emerging nano- and bio-engineering techniques that fabricate lymphoid organs are promising to provide an artificial environment for DCs and T cell priming. Thus, nanotechnology is a promising approach for DC-based therapeutics and can promote multifield development with great impact on clinical cancer treatment.

\section{AUTHOR CONTRIBUTIONS}

CQ, L-JY, and HC wrote the manuscript. HC provided advice both on the review writing and discussion.

\section{FUNDING}

This work was supported by Beijing Municipal Science \& Technology Commission (Z171100001717020).
Beltran, H., Beer, T. M., Carducci, M. A., De Bono, J., Gleave, M., Hussain, M., et al. (2011). New Therapies for Castration-Resistant Prostate Cancer: Efficacy and Safety. Eur. Urol. 60, 279-290. doi: 10.1016/j.eururo.2011.04.038

Berinstein, N. L., Karkada, M., Morse, M. A., Nemunaitis, J. J., Chatta, G., Kaufman, H., et al. (2012). First-in-man application of a novel therapeutic cancer vaccine formulation with the capacity to induce multi-functional $\mathrm{T}$ cell responses in ovarian, breast and prostate cancer patients. J. Trans. Med. 10, 156. doi: 10.1186/1479-5876-10-156

Bethke, K., Staib, F., Distler, M., Schmitt, U., Jonuleit, H., Enk, A. H., et al. (2002). Different efficiency of heat shock proteins (HSP) to activate human monocytes and dendritic cells: superiority of HSP60. J. Immunol. 169, 6141-6148. doi: 10.4049/jimmunol.169.11.6141

Binnewies, M., Roberts, E. W., Kersten, K., Chan, V., Fearon, D. F., Merad, M., et al. (2018). Understanding the tumor immune microenvironment (TIME) for effective therapy. Nat. Med. 24, 541-550. doi: 10.1038/s41591-018-0014-x

Brahmer, J., Reckamp, K. L., Baas, P., Crino, L., Eberhardt, W. E. E., Poddubskaya, E., et al. (2015). Nivolumab versus Docetaxel in Advanced Squamous-Cell NonSmall-Cell Lung Cancer. New Engl. J. Med. 373, 123-135. doi: 10.1056/ NEJMoa1504627

Butts, C., Socinski, M. A., Mitchell, P. L., Thatcher, N., Havel, L., Krzakowski, M., et al. (2014). Tecemotide (L-BLP25) versus placebo after chemoradiotherapy 
for stage III non-small-cell lung cancer (START): a randomised, double-blind, phase 3 trial. Lancet Oncol. 15, 59-68. doi: 10.1016/S1470-2045(13)70510-2

Chen, Q., Xu, L., Liang, C., Wang, C., Peng, R., and Liu, Z. (2016). Photothermal therapy with immune-adjuvant nanoparticles together with checkpoint blockade for effective cancer immunotherapy. Nat. Commun. 7, 13193. doi: $10.1038 /$ ncomms13193

Chen, W. S., Ouyang, J., Liu, H., Chen, M., Zeng, K., Sheng, J. P., et al. (2017). Black Phosphorus Nanosheet-Based Drug Delivery System for Synergistic Photodynamic/Photothermal/Chemotherapy of Cancer. Adv Mater 29, 1-7. doi: 10.1002/adma.201603864

Coffey, J. C., Wang, J. H., Smith, M. J. F., Bouchier-Hayes, D., Cotter, T. G., and Redmond, H. P. (2003). Excisional surgery for cancer cure: therapy at a cost. Lancet Oncol. 4, 760-768. doi: 10.1016/S1470-2045(03)01282-8

Cohen, C. J., Denkberg, G., Lev, A., Epel, M., and Reiter, Y. (2003). Recombinant antibodies with MHC-restricted, peptide-specific, T-cell receptor-like specificity: new tools to study antigen presentation and TCR-peptide-MHC interactions. J. Mol. Recog 16, 324-332. doi: 10.1002/jmr.640

Constantino, J., Gomes, C., Falcão, A., Cruz, M. T., and Neves, B. M. (2016). Antitumor dendritic cell-based vaccines: lessons from 20 years of clinical trials and future perspectives. Trans. Res. 168, 74-95. doi: 10.1016/j.trsl.2015.07.008

Costa, A., Kieffer, Y., Scholer-Dahirel, A., Pelon, F., Bourachot, B., Cardon, M., et al. (2018). Fibroblast Heterogeneity and Immunosuppressive Environment in Human Breast Cancer. Cancer Cell 33, 463-46+. doi: 10.1016/j.ccell.2018.01.011

Couzin-Frankel, J. (2013). Cancer immunotherapy. Science 342, 1432-1433. doi: 10.1126/science.342.6165.1432

Da Silva, C., Rueda, F., Löwik, C., Ossendorp, F., and Cruz, L. J. (2016). Combinatorial prospects of nano-targeted chemoimmunotherapy. Biomaterials 83, 308-320. doi: 10.1016/j.biomaterials.2016.01.006

Dadi, S., Chhangawala, S., Whitlock, B. M., Franklin, R. A., Luo, C. T., Oh, S. A., et al. (2016). Cancer immunosurveillance by tissue-resident innate lymphoid cells and innate-like T cells. Cell 164, 365-377. doi: 10.1016/j.cell.2016.01.002

De Koker, S., Cui, J., Vanparijs, N., Albertazzi, L., Grooten, J., Caruso, F., et al. (2016). Engineering Polymer Hydrogel Nanoparticles for Lymph NodeTargeted Delivery. Angewandte Chemie Int. Ed 55, 1334-1339. doi: 10.1002/ anie. 201508626

Delamarre, L., Pack, M., Chang, H., Mellman, I., and Trombetta, E. S. (2005). Differential Lysosomal Proteolysis in Antigen-Presenting Cells Determines Antigen Fate. Science 307, 1630. doi: 10.1126/science.1108003

Dellacherie, M. O., Li, A. W., Lu, B. Y., and Mooney, D. J. (2018). Covalent conjugation of peptide antigen to mesoporous silica rods to enhance cellular responses. Bioconjug Chem. 29, 733-741. doi: 10.1021/acs.bioconjchem.7b00656

Draube, A., Klein-Gonzalez, N., Mattheus, S., Brillant, C., Hellmich, M., Engert, A., et al. (2011). Dendritic cell based tumor vaccination in prostate and renal cell cancer: a systematic review and meta-analysis. PloS One 6, 1-11. doi: 10.1371/ journal.pone.0018801

Feng, B., Zhou, F. Y., Hou, B., Wang, D. G., Wang, T. T., Fu, Y. L., et al. (2018). Binary Cooperative Prodrug Nanoparticles Improve Immunotherapy by Synergistically Modulating Immune Tumor Microenvironment. Adv Mater 30, 1-10. doi: 10.1002/adma.201803001

Feng, X., Xu, W., Li, Z., Song, W., Ding, J., and Chen, X. (2019). Immunomodulatory Nanosystems. Adv Sci. 6, 1900101. doi: 10.1002/ advs. 201900101

Ghadersohi, A., and Sood, A. K. (2001). Prostate epithelium-derived Ets transcription factor mRNA is overexpressed in human breast tumors and is a candidate breast tumor marker and a breast tumor antigen. Clin. Cancer Res. 7, 2731-2738.

Goodwin, T. J., and Huang, L. (2017). Investigation of phosphorylated adjuvants co-encapsulated with a model cancer peptide antigen for the treatment of colorectal cancer and liver metastasis. Vaccine 35, 2550-2557. doi: 10.1016/ j.vaccine.2017.03.067

Granot, T., Senda, T., Carpenter, D. J., Matsuoka, N., Weiner, J., Gordon, C. L., et al. (2017). Dendritic Cells Display Subset and Tissue-Specific Maturation Dynamics over Human Life. Immunity 46, 504-515. doi: 10.1016/ j.immuni.2017.02.019

Guéry, L., and Hugues, S. (2015). Th17 Cell Plasticity and Functions in Cancer Immunity. BioMed. Res. Int. 2015, 314620. doi: 10.1155/2015/314620
Gulla, S. K., Rao, B. R., Moku, G., Jinka, S., Nimmu, N. V., Khalid, S., et al. (2019). In vivo targeting of DNA vaccines to dendritic cells using functionalized gold nanoparticles. Biomater Sci. 7, 773-788. doi: 10.1039/C8BM01272E

Guth, U., Myrick, M. E., Schotzau, A., Kilic, N., and Schmid, S. M. (2011). Drug switch because of treatment-related adverse side effects in endocrine adjuvant breast cancer therapy: how often and how often does it work? Breast Cancer Res. Treat 129, 799-807. doi: 10.1007/s10549-011-1668-y

Haanen, J. B. (2017). Converting cold into hot tumors by combining immunotherapies. Cell 170, 1055-1056. doi: 10.1016/j.cell.2017.08.031

Higano, C. S., Schellhammer, P. F., Small, E. J., Burch, P. A., Nemunaitis, J., Yuh, L., et al. (2009). Integrated Data From 2 Randomized, Double-Blind, PlaceboControlled, Phase 3 Trials of Active Cellular Immunotherapy With Sipuleucel-T in Advanced Prostate Cancer. Cancer 115, 3670-3679. doi: 10.1002/cncr.24429

Ho, N. I., Huis in 'T Veld, L. G. M., Raaijmakers, T. K., and Adema, G. J. (2018). Adjuvants Enhancing Cross-Presentation by Dendritic Cells: The Key to More Effective Vaccines? Front. Immunol. 9, 1-12. doi: 10.3389/fimmu.2018.02874

Housman, G., Byler, S., Heerboth, S., Lapinska, K., Longacre, M., Snyder, N., et al. (2014). Drug Resistance in Cancer: An Overview. Cancers 6, 1769-1792. doi: $10.3390 /$ cancers 6031769

Irvine, D. J., Swartz, M. A., and Szeto, G. L. (2013). Engineering synthetic vaccines using cues from natural immunity. Nat. Mater 12, 978-990. doi: 10.1038/ nmat 3775

Jennings, G. T., and Bachmann, M. F. (2007). Designing recombinant vaccines with viral properties: a rational approach to more effective vaccines. Curr. Mol. Med. 7, 143-155. doi: 10.2174/156652407780059140

Jiang, H., Wang, Q., and Sun, X. (2017). Lymph node targeting strategies to improve vaccination efficacy. J. Controlled Release 267, 47-56. doi: 10.1016/ j.jconrel.2017.08.009

Jiang, H., Wang, Q., Li, L., Zeng, Q., Li, H., Gong, T., et al. (2018). Turning the Old Adjuvant from Gel to Nanoparticles to Amplify CD8+ T Cell Responses. Adv Sci. 5, 1700426. doi: 10.1002/advs.201700426

Johnson, T. S., Mcgaha, T., and Munn, D. H. (2017). "Chemo-immunotherapy: role of indoleamine 2, 3-dioxygenase in defining immunogenic versus tolerogenic cell death in the tumor microenvironment," in Tumor Immune Microenvironment in Cancer Progression and Cancer Therapy and Cancer Therapy (Springer), 1036, 91-104.

June, C. H., O'connor, R. S., Kawalekar, O. U., Ghassemi, S., and Milone, M. C. (2018). CAR T cell immunotherapy for human cancer. Science 359, 1361-1365. doi: $10.1126 /$ science.aar6711

Kadowaki, N. (2009). The divergence and interplay between pDC and mDC in humans. Front. Biosci 14, 808-817. doi: 10.2741/3279

Kalinski, P., Muthuswamy, R., and Urban, J. (2013). Dendritic cells in cancer immunotherapy: vaccines and combination immunotherapies. Expert Rev. Vaccines 12, 285-295. doi: 10.1586/erv.13.22

Kantoff, P. W., Higano, C. S., Shore, N. D., Berger, E. R., Small, E. J., Penson, D. F., et al. (2010). Sipuleucel-T Immunotherapy for Castration-Resistant Prostate Cancer. New Engl. J. Med. 363, 411-422. doi: 10.1056/NEJMoa1001294

Kawai, T., and Akira, S. (2007). TLR signaling Semin Immunon. 19, 24-32.

Knudson, K. M., Hicks, K. C., Luo, X. L., Chen, J. Q., Schlom, J., and Gameiro, S. R. (2018). M7824, a novel bifunctional anti-PD-L1/TGF beta Trap fusion protein, promotes anti-tumor efficacy as monotherapy and in combination with vaccine. Oncoimmunology 7, 1-14. doi: 10.1080/2162402X.2018.1426519

Kongsted, P., Borch, T. H., Ellebaek, E., Iversen, T. Z., Andersen, R., Met, Ö, et al. (2017). Dendritic cell vaccination in combination with docetaxel for patients with metastatic castration-resistant prostate cancer: a randomized phase II study. Cytotherapy 19, 500-513. doi: 10.1016/j.jcyt.2017.01.007

Koppelmans, V., Breteler, M. M. B., Boogerd, W., Seynaeve, C., Gundy, C., and Schagen, S. B. (2012). Neuropsychological Performance in Survivors of Breast Cancer More Than 20 Years After Adjuvant Chemotherapy. J. Clin. Oncol. 30, 1080-1086. doi: 10.1200/JCO.2011.37.0189

Krook, J. E., Moertel, C. G., Gunderson, L. L., Wieand, H. S., Collins, R. T., Beart, R. W., et al. (1991). Effective surgical adjuvant therapy for high-risk rectal-carcinoma. New Engl. J. Med. 324, 709-715. doi: 10.1056/NEJM199103143241101

Kuai, R., Ochyl, L. J., Bahjat, K. S., Schwendeman, A., and Moon, J. J. (2017). Designer vaccine nanodiscs for personalized cancer immunotherapy. Nat. Mater 16, 489-496. doi: 10.1038/nmat4822 
Lövgren, T., Wolodarski, M., Edbäck, U., Martell, E., Markland, K., Nyström, M., et al. (2018). Abstract CT032: Adoptive T cell transfer combined with DC vaccination in patients with metastatic melanoma. Cancer Research 78. doi: 10.1158/1538-7445.AM2018-CT032

Le, D. T., Uram, J. N., Wang, H., Bartlett, B. R., Kemberling, H., Eyring, A. D., et al. (2015). PD-1 Blockade in Tumors with Mismatch-Repair Deficiency. New Engl. J. Med. 372, 2509-2520. doi: 10.1056/NEJMoa1500596

Leary, M., Heerboth, S., Lapinska, K., and Sarkar, S. (2018). Sensitization of Drug Resistant Cancer Cells: A Matter of Combination Therapy. Cancers 10, 1-18. doi: 10.3390/cancers 10120483

Leifer, C. A. (2017). Dendritic cells in host response to biologic scaffolds. Semin. Immunol. 29, 41-48. doi: 10.1016/j.smim.2017.01.001

Leitner, W. W., Ying, H., and Restifo, N. P. (1999). DNA and RNA-based vaccines: principles, progress and prospects. Vaccine 18, 765-777. doi: 10.1016/S0264410X(99)00271-6

Li, Y., Zeng, X., He, L., and Yuan, H. (2015). Dendritic cell activation and maturation induced by recombinant calreticulin fragment 39-272. Int. J. Clin. Exp. Med. 8, 7288.

Li, F., Chen, W.-L., You, B.-G., Liu, Y., Yang, S.-D., Yuan, Z.-Q., et al. (2016). Enhanced cellular internalization and on-demand intracellular release of doxorubicin by stepwise $\mathrm{pH}$-/reduction-responsive nanoparticles. ACS Appl. Mater Interfaces 8, 32146-32158. doi: 10.1021/acsami.6b09604

Lin, Y.-X., Wang, Y., Blake, S., Yu, M., Mei, L., Wang, H., et al. (2020). RNA Nanotechnology-Mediated Cancer Immunotherapy. Theranostics 10, 281. doi: 10.7150/thno. 35568

Lippert, T. H., Ruoff, H. J., and Volm, M. (2008). Intrinsic and acquired drug resistance in malignant tumors. Arzneimittelforschung-Drug Res. 58, 261-264. doi: 10.1055/s-0031-1296504

Liu, H. Y., Chen, D., Li, L. L., Liu, T. L., Tan, L. F., Wu, X. L., et al. (2011). Multifunctional Gold Nanoshells on Silica Nanorattles: A Platform for the Combination of Photothermal Therapy and Chemotherapy with Low Systemic Toxicity. Angewandte Chemie-International Ed 50, 891-895. doi: 10.1002/ anie. 201002820

Liu, Z., Jiang, W., Nam, J., Moon, J. J., and Kim, B. Y. S. (2018). Immunomodulating Nanomedicine for Cancer Therapy. Nano Lett. 18, 6655-6659. doi: 10.1021/acs.nanolett.8b02340

Lizotte, P., Wen, A., Sheen, M., Fields, J., Rojanasopondist, P., Steinmetz, N., et al. (2016). In situ vaccination with cowpea mosaic virus nanoparticles suppresses metastatic cancer. Nat. Nanotechnol 11, 295. doi: 10.1038/nnano.2015.292

Lu, J., Liu, X., Liao, Y.-P., Wang, X., Ahmed, A., Jiang, W., et al. (2018). Breast cancer chemo-immunotherapy through liposomal delivery of an immunogenic cell death stimulus plus interference in the IDO-1 pathway. ACS Nano 12, 11041-11061. doi: 10.1021/acsnano.8b05189

Lucky, S. S., Soo, K. C., and Zhang, Y. (2015). Nanoparticles in Photodynamic Therapy. Chem. Rev. 115, 1990-2042. doi: 10.1021/cr5004198

Lussier, D. M., and Schreiber, R. (2016). Cancer immunosurveillance: immunoediting. Immunity Patho. Tumors 4, 396-405. doi: 10.1016/B978-0-12-374279-7.17001-8

Macdonald, J. S., Smalley, S. R., Benedetti, J., Hundahl, S. A., Estes, N. C., Stemmermann, G. N., et al. (2001). Chemoradiotherapy after surgery compared with surgery alone for adenocarcinoma of the stomach or gastroesophageal junction. New Engl. J. Med. 345, 725-730. doi: 10.1056/NEJMoa010187

Manfredi, A. A., Capobianco, A., Esposito, A., De Cobelli, F., Canu, T., Monno, A., et al. (2008). Maturing Dendritic Cells Depend on RAGE for In Vivo Homing to Lymph Nodes. J. Immunol. 180, 2270. doi: 10.4049/jimmunol.180.4.2270

Martín-Fontecha, A., Lanzavecchia, A., and Sallusto, F. (2009). "Dendritic Cell Migration to Peripheral Lymph Nodes," in Dendritic Cells. Eds. G. Lombardi and Y. Riffo-Vasquez (Berlin, Heidelberg: Springer Berlin Heidelberg), 31-49.

Mathan, T. S. M., Textor, J., Skold, A. E., Reinieren-Beeren, I., Van Oorschot, T., Bruning, M., et al. (2017). Harnessing RNA sequencing for global, unbiased evaluation of two new adjuvants for dendritic-cell immunotherapy. Oncotarget 8, 19879-19893. doi: 10.18632/oncotarget.15190

Maude, S. L., Laetsch, T. W., Buechner, J., Rives, S., Boyer, M., Bittencourt, H., et al. (2018). Tisagenlecleucel in Children and Young Adults with B-Cell Lymphoblastic Leukemia. New Engl. J. Med. 378, 439-448. doi: 10.1056/ NEJMoa1709866

Mempel, T. R., Henrickson, S. E., and Von Andrian, U. H. (2004). T-cell priming by dendritic cells in lymph nodes occurs in three distinct phases. Nature 427, 154-159. doi: 10.1038/nature02238
Minton, K. (2014). IFN-dependent DC maturation. Nat. Rev. Immunol. 14, 67-67. doi: $10.1038 /$ nri3618

Morrison, D. C., and Kline, L. F. (1977). Activation of the classical and properdin pathways of complement by bacterial lipopolysaccharides (LPS). J. Immunol. $118,362-368$.

Murugaiyan, G., and Saha, B. (2009). Protumor vs Antitumor Functions of IL-17. J. Immunol. 183, 4169. doi: 10.4049/jimmunol.0901017

Nam, J., Son, S., Park, K. S., Zou, W., Shea, L. D., and Moon, J. J. (2019). Cancer nanomedicine for combination cancer immunotherapy. Nat. Rev. Mater 4, 398-414. doi: 10.1038/s41578-019-0108-1

Nicholson, L. B. (2016). The immune system. Essays Biochem. 60, 275-301. doi: 10.1042/ЕBC20160017

Ogasawara, M., Miyashita, M., and Ota, S. (2018). Vaccination of Urological Cancer Patients With WT1 Peptide-Pulsed Dendritic Cells in Combination With Molecular Targeted Therapy or Conventional Chemotherapy Induces Immunological and Clinical Responses. Ther. Apheresis Dialysis 22, 266-277. doi: 10.1111/1744-9987.12694

Paglia, P., Chiodoni, C., Rodolfo, M., and Colombo, M. P. (1996). Murine dendritic cells loaded in vitro with soluble protein prime cytotoxic $\mathrm{T}$ lymphocytes against tumor antigen in vivo. J. Exp. Med. 183, 317-322. doi: 10.1084/jem.183.1.317

Palma, M., Hansson, L., Mulder, T. A., Adamson, L., Näsman-Glaser, B., Eriksson, I., et al. (2018). Lenalidomide as immune adjuvant to a dendritic cell vaccine in chronic lymphocytic leukemia patients. Eur. J. Haematol 101, 68-77. doi: 10.1111/ejh.13065

Parvanian, S., Mostafavi, S. M., and Aghashiri, M. (2017). Multifunctional nanoparticle developments in cancer diagnosis and treatment. Sens. Biosensing Res. 13, 81-87. doi: 10.1016/j.sbsr.2016.08.002

Probst, H. C., Mccoy, K., Okazaki, T., Honjo, T., and Van Den Broek, M. (2005). Resting dendritic cells induce peripheral CD8+ T cell tolerance through PD-1 and CTLA-4. Nat. Immunol. 6, 280-286. doi: 10.1038/ni1165

Raucci, A., Palumbo, R., and Bianchi, M. E. (2007). HMGB1: a signal of necrosis. Autoimmunity 40, 285-289. doi: 10.1080/08916930701356978

Reck, M., Rodriguez-Abreu, D., Robinson, A. G., Hui, R. N., Csoszi, T., Fulop, A., et al. (2016). Pembrolizumab versus Chemotherapy for PD-L1-Positive NonSmall-Cell Lung Cancer. New Engl. J. Med. 375, 1823-1833. doi: 10.1056/ NEJMoa1606774

Reddy, S. T., Van Der Vlies, A. J., Simeoni, E., Angeli, V., Randolph, G. J., O’neil, C. P., et al. (2007). Exploiting lymphatic transport and complement activation in nanoparticle vaccines. Nat. Biotechnol. 25, 1159-1164. doi: 10.1038/nbt1332

Reichmuth, A. M., Oberli, M. A., Jaklenec, A., Langer, R., and Blankschtein, D. (2016). mRNA vaccine delivery using lipid nanoparticles. Ther. Deliv 7, 319334. doi: 10.4155/tde-2016-0006

Rescigno, M., Winzler, C., Delia, D., Mutini, C., Lutz, M., and RicciardiCastagnoli, P. (1997). Dendritic cell maturation is required for initiation of the immune response. J. Leukoc Biol. 61, 415-421. doi: 10.1002/jlb.61.4.415

Robert, C., Thomas, L., Bondarenko, I., O'day, S., Weber, J., Garbe, C., et al. (2011). Ipilimumab plus Dacarbazine for Previously Untreated Metastatic Melanoma. New Engl. J. Med. 364, 2517-2526. doi: 10.1056/NEJMoa1104621

Roden, R. B., and Stern, P. L. (2018). Opportunities and challenges for human papillomavirus vaccination in cancer. Nat. Rev. Cancer 18, 240. doi: 10.1038/ nrc.2018.13

Rogel, A., Popliker, M., Webb, C. G., and Oren, M. (1985). p53 cellular tumor antigen: analysis of mRNA levels in normal adult tissues, embryos, and tumors. Mol. Cell. Biol. 5, 2851-2855. doi: 10.1128/MCB.5.10.2851

Rothermundt, C. (2015). Management of side effects and adherence to oral cancer therapies in metastatic renal cell cancer. Oncol. Res. Treat 38, 17-17.

Rutella, S., Danese, S., and Leone, G. (2006). Tolerogenic dendritic cells: cytokine modulation comes of age. Blood 108, 1435-1440. doi: 10.1182/blood-2006-03006403

Sangtani, A., Nag, O. K., Field, L. D., Breger, J. C., and Delehanty, J. B. (2017). Multifunctional nanoparticle composites: progress in the use of soft and hard nanoparticles for drug delivery and imaging. Wiley Interdiscip. Rev: Nanomed Nanobiotechnol 9, e1466. doi: 10.1002/wnan.1466

Sau, S., Alsaab, H. O., Bhise, K., Alzhrani, R., Nabil, G., and Iyer, A. K. (2018). Multifunctional nanoparticles for cancer immunotherapy: a groundbreaking approach for reprogramming malfunctioned tumor environment. J. Controlled Release 274, 24-34. doi: 10.1016/j.jconrel.2018.01.028 
Sauer, R., Becker, H., Hohenberger, W., Rodel, C., Wittekind, C., Fietkau, R., et al. (2004). Preoperative versus postoperative chemoradiotherapy for rectal cancer. New Engl. J. Med. 351, 1731-1740. doi: 10.1056/NEJMoa040694

Schnurr, M., Then, F., Galambos, P., Scholz, C., Siegmund, B., Endres, S., et al. (2000). Extracellular ATP and TNF- $\alpha$ synergize in the activation and maturation of human dendritic cells. J. Immunol. 165, 4704-4709. doi: 10.4049/jimmunol.165.8.4704

Segura, E., and Villadangos, J. A. (2009). Antigen presentation by dendritic cells in vivo. Curr. Opin. Immunol. 21, 105-110. doi: 10.1016/j.coi.2009.03.011

Segura, E., Touzot, M., Bohineust, A., Cappuccio, A., Chiocchia, G., Hosmalin, A., et al. (2013). Human Inflammatory Dendritic Cells Induce Th17 Cell Differentiation. Immunity 38, 336-348. doi: 10.1016/j.immuni.2012.10.018

Sharma, M. D., Rodriguez, P. C., Koehn, B. H., Baban, B., Cui, Y., Guo, G., et al. (2018). Activation of p53 in immature myeloid precursor cells controls differentiation into Ly6c+ CD103+ monocytic antigen-presenting cells in tumors. Immunity 48, 91-106. e106. doi: 10.1016/j.immuni.2017.12.014

Sil, P., Zhao, F., Muse, G. W., Wong, S.-W., Kolb, J. P., Degraff, L. M., et al. (2019). Non-canonical autophagy in dendritic cells restricts cross-presentation and anti-tumor immunity. bioRxiv 789867. doi: 10.1101/789867

Simmons, D. P., Wearsch, P. A., Canaday, D. H., Meyerson, H. J., Liu, Y. C., Wang, Y., et al. (2012). Type I IFN Drives a Distinctive Dendritic Cell Maturation Phenotype That Allows Continued Class II MHC Synthesis and Antigen Processing. J. Immunol. 188, 3116. doi: 10.4049/jimmunol.1101313

Smith, G., Liu, Y., Tian, J.-H., Massare, M. J., Boddapati, S., Shane, E., et al. (2018). Vaccine compositions having improved stability and immunogenicity. WO/ $2017 / 041100$

Smyth, M. J., Dunn, G. P., and Schreiber, R. D. (2006). Cancer immunosurveillance and immunoediting: the roles of immunity in suppressing tumor development and shaping tumor immunogenicity. $A d v$. Immunol. 90, 1-50. doi: 10.1016/S0065-2776(06)90001-7

Speiser, D. E., Schwarz, K., Baumgaertner, P., Manolova, V., Devevre, E., Sterry, W., et al. (2010). Memory and Effector CD8 T-cell Responses After Nanoparticle Vaccination of Melanoma Patients. J. Immunother 33, 848-858. doi: 10.1097/ CJI.0b013e3181f1d614

Thomas, R., and Lipsky, P. E. (1996). Dendritic cells: origin and differentiation. Stem Cells 14, 196-206. doi: 10.1002/stem.140196

Van Herck, S., Deswarte, K., Nuhn, L., Zhong, Z., Portela Catani, J. P., Li, Y., et al. (2018). Lymph-node-targeted immune activation by engineered block copolymer amphiphiles-TLR7/8 agonist conjugates. J. Am. Chem. Soc. 140, 14300-14307. doi: 10.1021/jacs.8b08595

Vansteenkiste, J. F., Cho, B., Vanakesa, T., De Pas, T., Zielinski, M., Kim, M. S., et al. (2014). Magrit, a Double-Blind, Randomized, Placebo-Controlled Phase III Study to Assess the Efficacy of the Recmage-A3 + As15 Cancer Immunotherapeutic As Adjuvant Therapy in Patients with Resected MageA3-Positive Non-Small Cell Lung Cancer (Nsclc). Ann. Oncol. 25, iv409. doi: 10.1093/annonc/mdu347.1

Wang, H., and Mooney, D. J. (2018). Biomaterial-assisted targeted modulation of immune cells in cancer treatment. Nat. Mater 17, 761-772. doi: 10.1038/ s41563-018-0147-9

Wang, Y., Lin, Y. X., Qiao, Z. Y., An, H. W., Qiao, S. L., Wang, L., et al. (2015). Self-Assembled Autophagy-Inducing Polymeric Nanoparticles for Breast Cancer Interference In-Vivo. Adv Mater 27, 2627-2634. doi: 10.1002/ adma.201405926

Wang, S., Huang, P., and Chen, X. (2016). Hierarchical targeting strategy for enhanced tumor tissue accumulation/retention and cellular internalization. Adv Mater 28, 7340-7364. doi: 10.1002/adma.201601498

Wang, Y., Lin, Y.-X., Qiao, S.-L., An, H.-W., Ma, Y., Qiao, Z.-Y., et al. (2017). Polymeric nanoparticles enable reversing macrophage in tumor microenvironment for immunotherapy. Biomaterials 112, 3e163.

Wang, Y., Lin, Y.-X., Wang, J., Qiao, S.-L., Liu, Y.-Y., Dong, W.-Q., et al. (2019a). In Situ Manipulation of Dendritic Cells by an Autophagy-Regulative Nanoactivator Enables Effective Cancer Immunotherapy. ACS Nano 13, 7568-7577. doi: 10.1021/acsnano.9b00143
Wang, Y., Lin, Y. X., Qiao, S. L., Wang, J., and Wang, H. (2019b). Progress in Tumor-Associated Macrophages: From Bench to Bedside. Adv Biosyst. 3, 1800232. doi: 10.1002/adbi.201800232

Wen, R., Umeano, A. C., Kou, Y., Xu, J., and Farooqi, A. A. (2019). Nanoparticle systems for cancer vaccine. Nanomedicine 14, 627-648. doi: 10.2217/nnm-2018-0147

Williams, J. W., Tjota, M. Y., Clay, B. S., Vander Lugt, B., Bandukwala, H. S., Hrusch, C. L., et al. (2013). Transcription factor IRF4 drives dendritic cells to promote Th2 differentiation. Nat. Commun. 4, 1-12. doi: 10.1038/ncomms3990

Wolf, G. T. (1991). Induction chemotherapy plus radiation compared with surgery plus radiation in patients with advanced laryngeal-cancer. New Engl. J. Med. 324, 1685-1690. doi: 10.1056/NEJM199106133242402

Wongso, D., Dong, J., Ueda, H., and Kitaguche, T. (2017). Flashbody: A Next Generation Fluobody with Fluorescence Intensity Enhanced by Antigen Binding. Anal Chem. 89, 6719-6725. doi: 10.1021/acs.analchem.7b00959

Yu, H., Yang, Y., Jiang, T., Zhang, X., Zhao, Y., Pang, G., et al. (2019). Effective Radiotherapy in Tumor Assisted by Ganoderma lucidum PolysaccharideConjugated Bismuth Sulfide Nanoparticles through Radiosensitization and Dendritic Cell Activation. ACS Appl. Mater Interfaces 11, 27536-27547. doi: 10.1021/acsami.9b07804

Zehn, D., Cohen, C. J., Reiter, Y., and Walden, P. (2004). Extended presentation of specific MHC-peptide complexes by mature dendritic cells compared to other types of antigen-presenting cells. Eur. J. Immunol. 34, 1551-1560. doi: 10.1002/ eji.200324355

Zhang, N., and Bevan, M. J. (2011). CD8+ T cells: foot soldiers of the immune system. Immunity 35, 161-168. doi: 10.1016/j.immuni.2011.07.010

Zhang, R., Smith, J. D., Allen, B. N., Kramer, J. S., Schauflinger, M., and Ulery, B. D. (2018). Peptide Amphiphile Micelle Vaccine Size and Charge Influence the Host Antibody Response. ACS Biomater Sci. Eng. 4, 2463-2472. doi: 10.1021/ acsbiomaterials.8b00511

Zhang, L., Wu, S., Qin, Y., Fan, F., Zhang, Z., Huang, C., et al. (2019). Targeted Codelivery of an Antigen and Dual Agonists by Hybrid Nanoparticles for Enhanced Cancer Immunotherapy. Nano Lett. 19, 4237-4249. doi: 10.1021/ acs.nanolett.9b00030

Zhao, P. F., Wang, Y. H., Kang, X. J., Wu, A. H., Yin, W. M., Tang, Y. S., et al. (2018). Dual-targeting biomimetic delivery for anti-glioma activity via remodeling the tumor microenvironment and directing macrophagemediated immunotherapy. Chem. Sci. 9, 2674-2689. doi: 10.1039/C7SC04853J

Zhong, L. L., Zhao, Y. L., Zhang, K., Li, X., Cui, R. J., and Yang, W. (2017). Recent advances of immune checkpoint in breast cancer. Biomed. Research-India 28, 7268-7273. doi: 10.1186/s12916-019-1326-5

Zhong, X., Zhang, Y., Tan, L., Zheng, T., Hou, Y., Hong, X., et al. (2019). An aluminum adjuvant-integrated nano-MOF as antigen delivery system to induce strong humoral and cellular immune responses. J. Controlled Release 300, 81-92. doi: 10.1016/j.jconrel.2019.02.035

Zhou, Q., Zhang, Y., Du, J., Li, Y., Zhou, Y., Fu, Q., et al. (2016). Different-sized gold nanoparticle activator/antigen increases dendritic cells accumulation in liver-draining lymph nodes and CD8+ T cell responses. ACS Nano 10, 26782692. doi: $10.1021 /$ acsnano. 5 b07716

Zupančič, E., Curato, C., Paisana, M., Rodrigues, C., Porat, Z., Viana, A. S., et al. (2017). Rational design of nanoparticles towards targeting antigen-presenting cells and improved T cell priming. J. Controlled Release 258, 182-195. doi: 10.1016/j.jconrel.2017.05.014

Conflict of Interest: The authors declare that the research was conducted in the absence of any commercial or financial relationships that could be construed as a potential conflict of interest.

Copyright (๑) 2020 Qian, Yang and Cui. This is an open-access article distributed under the terms of the Creative Commons Attribution License (CC BY). The use, distribution or reproduction in other forums is permitted, provided the original author(s) and the copyright owner(s) are credited and that the original publication in this journal is cited, in accordance with accepted academic practice. No use, distribution or reproduction is permitted which does not comply with these terms. 